\section{Mean ergodic theorem in locally convex linear topological spaces}

by

M. ALTMAN (Warszawa).

Kosaku Yosida has proved the following generalization of the mean ergodic theorem of J. v. NEUMANN:

Let $T$ be a linear transformation which maps a Banach space into itself. Let us further assume that and

$1^{0}$ the sequence $\left\{T^{n}(x)\right\}(n=1,2, \ldots)$ is bounded for any element $x$

$2^{0}$ the transformation $T$ is weakly completely continuous.

Under these assumptions, the sequence

$$
\left\{\frac{1}{n} \sum_{i=0}^{n-1} T^{i}(x)\right\}
$$

converges strongly to a point $y$, and $T(y)=y$.

Using the same method as in the paper [2] we can prove the above theorem for locally convex linear topological spaces.

Let $\mathfrak{X}$ be a locally convex linear topological space ${ }^{1}$ ) and let $\mathfrak{X}$ be the space of all linear functionals defined in $\mathfrak{X}$.

Definition 1. A sequence of elements $\left\{x_{n}\right\}$ is said to be weakly convergent to an element $x$ if for any $X_{\epsilon} \overrightarrow{\mathfrak{X}}$ the sequence $\left\{X\left(x_{n}\right)\right\}$ converges to $X(x)$.

Definition 2. A transformation $T$ is said to be weakly completely continuous if there exists a neighbourhood $O$ of zero such that the image $T(O)$ is sequentially weakly compact, i. e. any infinite part contains a sequence weakly convergent to some element of the space.

It is known (see [2]) that the space $\mathfrak{X}$ is isomorphic with a certain $\left(B_{a}^{*}\right)$-space in which is defined a class of pseudonorms $|x|_{\vartheta}$, where

1) Concerning the definition see [2].

$\vartheta \epsilon \Phi$. The system of neighbourhoods of zero consists of the sets of all elements $x \in \mathfrak{X}$ such that $|x|_{\theta_{i}}<\varepsilon(i=1,2, \ldots, k), \varepsilon>0$. ( $\Phi$ is an abstract set and $\overline{\bar{\Phi}}=\kappa_{\alpha}$ ).

The sequence $\left\{x_{n}\right\}$ is said to be convergent to zero if $\left|x_{n}\right|_{\vartheta} \rightarrow 0$ for any $\vartheta \epsilon \Phi$.

Definition 3. A set $Z \subset \mathfrak{X}$ is said to be bounded if for any sequence $\left\{x_{n}\right\} \subset Z$ and for every arbitrary sequence of numbers $\lambda_{n}$ convergent to zero we have $\lambda_{n} x_{n} \rightarrow 0$, when $n \rightarrow \infty$.

Let $T$ be a linear ${ }^{2}$ ) weakly completely continuous transformation which maps the neighbourhood of zero $O$ into a sequentially weakly compact set. Then there exists a neighbourhood of zero $V$ such that $V \subset O$, where $V$ is defined by the inequalities $|x|_{\theta_{i}}<\varepsilon$ $(i=1,2, \ldots, k)$. We define the pseudonorm $|x|=\sup _{i}|x|_{\vartheta_{i}}$. Then the image of any set bounded in this pseudonorm is sequentially weakly compact and therefore bounded in the sense of the definition 3 . Hence it appears the following

Lemma. If $T$ is a linear weakly completely continuous transformation, then for every $\vartheta$ there exists a constant $\boldsymbol{M}_{\vartheta}$ such that $|T(x)|_{\vartheta} \leqslant M_{\vartheta}|x|$.

The proof is the same as in [2] (lemma 1).

Theorem. Let $T$ be a linear transformation which maps a locally convex linear topological space $\mathfrak{X}$ into itself. Let us further assume that

$1^{0}$ the sequence $\left\{T^{n}(x)\right\}(n=1,2, \ldots)$ is bounded for any $x \in \mathfrak{X}$,

$2^{0}$ the transformation $T$ is weakly completely continuous.

Under these assumptions, the sequence

$$
\left\{\frac{1}{n} \sum_{i=0}^{n-1} T^{i}(x)\right\}
$$

converges for any $x \in \mathfrak{X}$ to a point $y$, and $T(y)=y$.

Proof. We divide the space $\mathfrak{X}$ into classes and we say that $x_{1}$ and $x_{2}$ belong to the same class $\mathfrak{x}$, if $\left|x_{1}-x_{2}\right|=0$. The set of all elements $x \epsilon \mathfrak{X}$ such that $|x|=0$ constitutes the zero class. We denote by $\mathfrak{X}^{*}$ the obtained quotient space which is a Banach space with the norm $|\mathfrak{x}|=|x|$.

2) We consider the continuity in the sense of Heine, i. e. $x_{n} \rightarrow 0$ implies that $T\left(x_{n}\right)$ converges to zero. 
The transformation $y=T(x)$ defines a transformation $\mathfrak{y}=\mathfrak{I}(\mathfrak{x})$ in the space $\mathfrak{X}^{*}$, where $x \in \mathfrak{X}, y \in \mathfrak{y}$. It follows from the lemma that $\mathfrak{I}$ is a linear transformation. It is obvious that $\mathfrak{I}$ is a weakly completely continuous transformation.

We denote by $\mathfrak{X}^{\prime}$ the completion of the space $\mathfrak{X}^{*}$. The transformation $\mathfrak{I}$ can be extended on the whole space $\mathfrak{X}^{\prime}$. We shall show that the range of this extension is contained in $\mathfrak{X}^{*}$. Let $\mathfrak{x}$ be an arbitrary element of $\mathfrak{X}^{\prime}$. There exists a sequence $\left\{\mathfrak{x}_{n}\right\} \subset \mathfrak{X}^{*}$ such that $\mathfrak{x}_{n} \rightarrow \mathfrak{x}$. Since the sequence $\left\{\mathfrak{x}_{n}\right\}$ is convergent and therefore bounded, the sequence $\mathfrak{I}\left(\mathfrak{x}_{n}\right)$ is weakly compact in $\mathfrak{X}^{*}$ and convergent to $\mathfrak{y} \epsilon \mathfrak{X}^{*}$, hence $\mathfrak{y}=\mathfrak{I}(\mathfrak{x})$. From the condition $1^{0}$ it follows that the sequence $\left\{\mathfrak{I}^{n}(\mathfrak{x})\right\}$ is bounded for any $\mathfrak{x} \in \mathfrak{X}^{*}$. Since the range of the transformation $\mathfrak{I}$ is contained in $\mathfrak{X}^{*}$, the sequence $\left\{\mathfrak{I}^{n}(\mathfrak{x})\right\}$ is bounded for any $\mathfrak{x} \in \mathfrak{X}^{\prime}$, and the theorem of Kosaku Yosida is valid for the transformation $\mathfrak{I}$.

Consider now the sequence

$$
\left\{\frac{1}{n} \sum_{i=0}^{n-1} T^{i}(x)\right\}
$$

The sequence

$$
\left\{\frac{1}{n} \sum_{i=0}^{n-1} \mathfrak{I}^{i}(\mathfrak{x})\right\}, \quad \text { where } \quad x \in \mathfrak{X},
$$

converges to some element $\mathfrak{y} \epsilon \mathfrak{X}^{\prime}$, and $\mathfrak{y}=\mathfrak{T}(\mathfrak{y}) \epsilon \mathfrak{X}^{*}$. On account of the lemma and since

$$
\left|\frac{1}{n} \sum_{i=0}^{n-1} T^{i}(x)-\bar{y}\right|=\left|\frac{1}{n} \sum_{i=0}^{n-1} \mathfrak{I}^{i}(\mathfrak{x})-\mathfrak{y}\right|, \quad \text { where } \bar{y} \in \mathfrak{y},
$$

the sequence

$$
\left\{T\left(\frac{1}{n} \sum_{i=0}^{n-1} T^{i}(x)\right)\right\}=\left\{\frac{1}{n} \sum_{i=1}^{n} T^{i}(x)\right\}
$$

converges to $y=T(\bar{y})$. Since

$$
\frac{1}{n} \sum_{i=0}^{n-1} T^{i}(x)=\frac{1}{n} x+\sum_{i=1}^{n} T^{i}(x)-\frac{1}{n} T^{n}(x),
$$

the sequences $\{x / n\}$ and $\left\{T^{n}(x) / n\right\}$ converge to 0 , the sequence

$$
\left\{\frac{1}{n} \sum_{i=0}^{n-1} T^{i}(x)\right\}
$$

converges to $y$. Then the sequence

$$
\left\{\frac{1}{n} \sum_{i=1}^{n} T^{i}(x)\right\}
$$

converges to $T(y)$. Since

$$
\frac{1}{n} \sum_{i=1}^{n} T^{i}(x)=\frac{1}{n} \sum_{i=0}^{n-1} T^{i}(x)+\frac{1}{n} T^{n}(x)-\frac{1}{n} x,
$$

we have $T(y)=y$

\section{References.}

[1] Kosaku Yosida, Mean ergodic theorem in Banach spaces, Proc. ImpAcad. Japan 14 (1938), p. $292-294$.

[2] M. Altman, On linear functional equations in locally convex $7 i$ near topological spaces, Studia Mathematica, this volume.

(Reçu par la Rédaction le 16. 6. 1952) 